\title{
Biased Brownian motion as the operating principle for microscopic engines
}

\author{
Martin Bier, R. Dean Astumian \\ Department of Surgery, MC 6035, University of Chicago, 5841 South Maryland Avenue Chicago, IL 60637, USA
}

Received 28 December 1994; in revised form 16 January 1995

\begin{abstract}
The hydrolysis of adenosine triphosphate (ATP) has been shown to drive the motion of motor proteins along a biopolymer. These motor proteins are the smallest engines known and, in the absence of an ATP-to-adenosine diphosphate chemical potential, they execute Brownian motion. Therefore, it is reasonable to imagine that the energy released in ATP hydrolysis is used to bias, or rectify, Brownian motion in one direction. In this paper, we show, in terms of Fokker-Planck equations that we solve analytically, how a net flow can occur along a periodic potential, provided that this potential has an anisotropy and that there is an energy input. We work out two cases: one case where the energy input comes from a fluctuation of the periodic potential in time and one case where a variation of temperature within a period is maintained. An interesting feature of these systems is that they need "the correct amount" of thermal noise. Without thermal noise or with too much thermal noise, no net flow occurs and, in this sense, the systems we discuss are one more example of the lately much discussed phenomenon of stochastic resonance.
\end{abstract}

Keywords: ATP hydrolysis; Brownian motion; Fokker-Planck equations; Heat engine; Stochastic resonance

\section{Introduction}

An engine is a "machine for converting any of various forms of energy into mechanical force and motion" [1]. Macroscopic combustion engines work by initiating a chemical reaction and subsequently letting the expansion and/or heat do work. This can be understood and described in terms of thermodynamics.

In living cells, motor proteins turn adenosine triphosphate (ATP) into adenosine diphosphate (ADP) and use the released energy to move in one direction along a biopolymer. As such, they are responsible for muscle action and they also take care of much of the transport within a cell [2]. The size of a biological cell, however, does not allow for a combustion engine to provide the driving mechanism for motor proteins. The proteins and polymers involved operate on a length-scale that is comparable with that to which Robert Brown referred in 1826 , when he observed how pollen particles make apparently random motions. In the Brownian realm, thermodynamics is no longer the appropriate framework of description and a mechanism different from Carnot cycling has to be responsible for the transduction of energy: a Langevin equation (or, equivalently, a Fokker-Planck equation) [3] must be employed to describe how a single molecule acts in response to the combined effect of a force and the random "kicks" of the molecules of the surrounding medium.

Many descriptions and models for motor proteins have viewed the protein as a system of levers and springs, and have relied on the quantitatively unspecifiable notion of a "conformational change" to describe how forces and tensions change as ATP is bound, converted and released as ADP and inorganic phosphate [4]. We will consider a more basic level and view the protein as a Brownian particle moving along a line. Our approach can lead to actual estimates for the speed and efficiency of a motor protein.

Unlike a macroscopic engine, a motor protein is still making random Brownian (but unbiased) motions along the biopolymer in the absence of its "fuel". For microscopic engines, the idea is to set up a mechanism where Brownian motion is rectified. Rectifiers are well known in the macroscopic world and are commonly employed in engineering. Electronic rectifiers are called diodes and give a current in response to a voltage that is higher in one direction than in the other direction. Mechanical rectifiers, otherwise known as "ratchets", are common in bicycles, carjacks and Sears screwdrivers. 
In his Lectures on Physics [5], Richard Feynman presented a microscopic ratchet-and-pawl device with an angular velocity-torque characteristic that was identical to the current-voltage characteristic of a diode. It is obvious that the response of a diode to a zero average a.c. voltage is a current, the average of which is not zero. As early as 1950 , Brillioun discussed the paradox that arises if rectifiers are taken into the microscopic realm [6]. Equilibrium fluctuations are always present at non-zero temperatures and there is the question of what happens when these are rectified. If the rectifier were to operate so as to produce net displacement or current (i.e. work) out of equilibrium fluctuations, then we would extract work out of a system in equilibrium and face a serious violation of the second law of thermodynamics. In the Lectures on Physics, Feynman showed in a very elegant manner that, upon close inspection, his ratchet-and-pawl device could not do any work in an equilibrium environment and would work similarly to a perfect Carnot engine if the vanes and the ratchet were kept in equilibrium reservoirs at different temperatures.

Rectifying Brownian motion in an equilibrium environment would be in obvious violation of the second law of thermodynamics. However, we will show here how, in a non-equilibrium environment, the dissipation of various forms of energy (chemical or heat) can result in the biasing of Brownian motion. Our approach is one of very basic physics. The only type of equations that we use are Fokker-Planck equations, in the overdamped limit for a stationary probability density distribution, and our treatment will be analytical.

\section{A chemical engine}

With our first example, we show how the fluctuations brought about by a reaction proceeding down its chemical potential gradient can make a motor protein move along a biopolymer and how the protein comes to a net standstill at chemical equilibrium.

Let us assume that $U^{+}$is the potential between the motor protein-ATP complex and the biopolymer and that $U^{-}$is the potential between just the motor protein and the biopolymer. $U(x)$ is a measure of how strongly the motor protein is pinned to the biopolymer at $x . U^{+}(x)$ and $U^{-}(x)$ must be periodic in $x$ but are otherwise arbitrary. We scale the length of this period to be one (Fig. 1).

For a very small protein in the intracellular solution, it is realistic to assume that we are in the overdamped limit, where the velocity is proportional to the instantaneous force. The equation of motion is a Langevin equations, i.e.

$$
\beta \dot{x}=-\partial_{x} U(x, t)+(2 \beta k T)^{1 / 2} \xi(t)
$$

where $\beta$ is the coefficient of friction and $\xi(t)$ represents white noise. After absorbing $\beta$ in the time-scale and

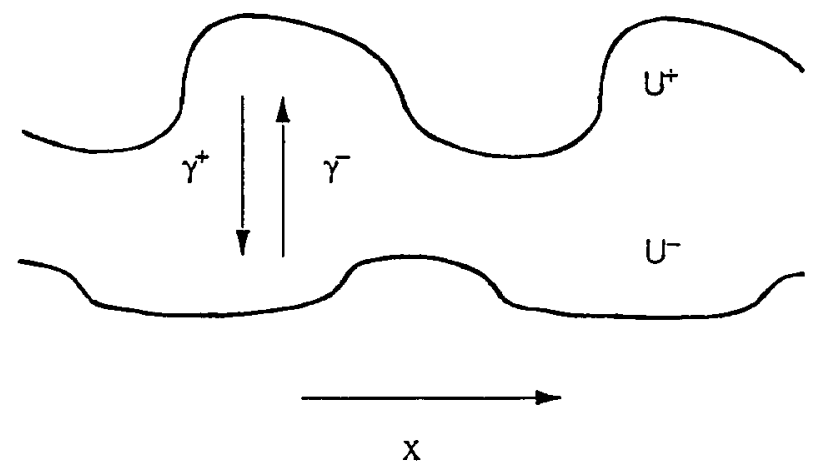

Fig. 1. Schematic diagram of chemical engine system.

taking $k T$ as the unit of energy, the stationary joint probability density $P^{ \pm}(x)$ for the particle to be at $x$ and the potential to be in the plus or minus mode is given by the following Fokker-Planck equations [3]:

$\partial_{x}^{2} P^{+}+\partial_{x}\left(U_{x}^{+} P^{+}\right)-\gamma^{+} P^{+}+\gamma^{-} P^{-}=0$

$\partial_{x}^{2} P^{-}+\partial_{x}\left(U_{x}^{-} P^{-}\right)+\gamma^{+} P^{+}-\gamma^{-} P^{-}=0$,

where $U_{x}$ represents the derivative of $U$ with respect to $x$. The $\gamma P$ terms arise from the flipping, the $\partial_{x}\left(U_{x} P\right)$ terms represent the force resulting from the potential, and the $\partial_{x}^{2} P$ terms describe the effect of diffusion and are a consequence of the $\xi(t)$ term in the Langevin equation [3].

At equilibrium, the ratio of the transition rates at any point $x$ depends only on the energy difference $\left(U^{+}-U^{-}\right)$ at that point, i.e.

$\frac{\gamma^{+}(x)}{\gamma^{-}(x)}=\exp \left(U^{+}-U^{-}\right)$

This leads to a Boltzmann distribution for $P^{+}$and $P^{-}$, i.e.

$\frac{P^{+}(x)}{P^{-}(x)}=\exp \left(U^{-}-U^{+}\right)$,

Thus, we have

$\gamma^{+}(x) P^{+}(x)-\gamma^{-}(x) P^{-}(x)=0$

and the last two terms in both Eqs. (2a) and (2b) cancel out. The remaining part of the system is solved by

$P^{ \pm}=C \exp \left(-U^{ \pm}\right)$

which is easily seen to lead to

$J^{ \pm}=\left(-U_{x}^{ \pm}-\partial_{x}\right) P^{ \pm}=0$

Therefore, at equilibrium, we obtain a consistent picture with a Boltzmann distribution, i.e. detailed balance and no flux.

However, if a different ratio of transition rates $\gamma^{+}$and $\gamma^{-}$is "forced upon" the system, i.e.

$\frac{\gamma^{+}(x)}{\gamma^{-}(x)} \neq \exp \left(U^{+}-U^{-}\right)$ 
then the transition rates between the positive and negative potential work against a Boltzmann distribution between the positive and negative potential. The diffusion along each of the separate potentials "strives" toward a Boltzmann distribution and a flow along $x$ in both $U^{+}$and $U^{-}$ will occur. Thus, the breakdown of detailed balance (just as many transitions in the plus to minus direction as in the minus to plus direction) between the positive and negative potentials at a point $x$ (i.e. a net flux between the two at $x$ ) causes a breakdown of detailed balance along the $x$ axis, so giving the possibility of a net flow along the $x$ direction.

The flux is maximal if the flipping time between the potentials is of the same order of magnitude as the relaxation time for diffusion to a Boltzmann distribution on $U^{ \pm}$. If the potential fluctuates too fast, then the diffusion along the $x$ coordinate can never get "started" and the system will simply create a stationary Boltzmann distribution on the average potential

$U_{\mathrm{avg}}=\left(\gamma^{+}+\gamma^{-}\right)\left(\frac{U^{-}}{\gamma^{-}}+\frac{U^{+}}{\gamma^{+}}\right)$

For a potential that fluctuates very slowly, the distribution will have the time after every flip to relax fully to either $U^{+}$or $U^{-}$and, in this low frequency realm, the flux is directly proportional to the fluctuation rate.

Obtaining $\gamma^{+}$and $\gamma^{-}$terms that are roughly constant and almost independent of $x$ can be achieved by implementing a chemical potential gradient (in most biological instances, this means an ATP-ADP potential) into the system, indicated in Fig. 2. If the ATP-ADP chemical potential is very high, then almost all the transitions will be the binding of ATP and the dissociation of ADP. The rate constants for the binding and dissociation of ATP and ADP respectively must obey the following equations:

$$
\begin{aligned}
& \frac{k_{1}^{-}[\mathrm{ATP}]}{k_{1}^{+}}=\exp \left(U^{-}-U^{+}\right) \exp \left(\Delta G_{1}\right) \\
& \frac{k_{2}^{-}[\mathrm{ADP}]\left[P_{\mathrm{i}}\right]}{k_{2}^{+}}=\exp \left(U^{-}-U^{+}\right) \exp \left(\Delta G_{2}\right)
\end{aligned}
$$

where $P_{\mathrm{j}}$ is inorganic phosphate and $\Delta G_{1}$ and $\Delta G_{2}$ are the position-independent free energy changes of binding

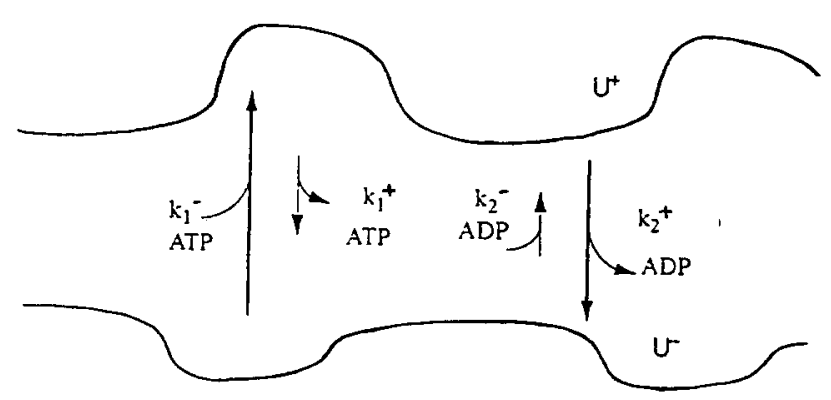

Fig. 2. Diagram showing chemical potential gradient in chemical engine system.
$\mathrm{ATP}$ and $\mathrm{ADP}+\mathrm{P}_{\mathrm{i}}$ to the motor respectively. The total energy released by ATP hydrolysis is $\Delta G_{\mathrm{ATP}}=\Delta G_{1}-$ $\Delta G_{2} . U^{+}$and $U^{-}$are functions of $x$, so the ratios $k_{1}^{-} / k_{1}^{+}$ and $k_{2}^{-} / k_{2}^{+}$are still dependent on $x$. However, it is possible to "hide" this $x$ dependence in the "unused" $k_{1}^{+}$ and $k_{2}^{-}$transitions, effectively creating a situation with position-independent transition rates. That the fluctuations are non-equilibrium is a necessary condition for net flux but by no means is a sufficient condition.

Because of continuity, i.e. $\partial_{t} P=\partial_{x} J$, a stationary solution implies a constant flux that is independent of $x$ and which can be worked out as

$J=J^{+}(x)+J^{-}(x)$

The power output of the engine can be derived as follows. Let us take $J$ as the average velocity $v$ of a Brownian particle; the power output is $F v$, where $F$ is the force necressary to keep the flow going - this force originates from friction and equals $\beta v$, but $\beta$ has been scaled to unity, so we end up with a power output $P_{\text {out }}=J^{2}$. The power input can be obtained by integrating the product of the probability density at $x$, the transition rate away from $x$ and the energy gained or lost in the transition, i.e.

$P_{\text {in }}=\int_{0}^{1}\left(\gamma^{+} P^{+}-\gamma^{-} P^{-}\right)\left(U^{-}-U^{+}\right) \mathrm{d} x$

It should be noted that the $\left(\gamma^{+}(x) P^{+}(x)-\gamma^{-}(x) P^{-}(x)\right)$ term is zero everywhere at equilibrium, which guarantees that the system is not powered at equilibrium.

The terms "white noise" and "equilibrium fluctuations" are commonly taken to be synonymous. Our system, however, reveals very clearly that these are two different things. Strictly defined, noise is white if it has a flat frequency spectrum but, for practical purposes, noise is white if most of its frequency spectrum is at frequencies much higher than the characteristic inverse time-scales of the system of interest [3]. In our set-up, we effectively have white non-equilibrium noise if we have a constant $\gamma^{+}=\gamma^{-}=\gamma$ and we go to the high frequency limit $\gamma \rightarrow \infty$. All we need for equilibrium noise is to satisfy Eq. (3), which fixes the ratio of $\gamma^{+}$and $\gamma^{-}$but leaves a degree of freedom with which we can place our noise at any inverse time-scale above, at or below the characteristic inverse times of the system. Thus, equilibrium noise can be arbitrarily slow. Of course, neither the white nor the equilibrium noise brings about a flux.

What characteristics of the noise constitute necessary and/or sufficient conditions for a flux in either the forward or backward direction is presently the topic of much research [7,8]. Doering, et al. [7] have recently published results that they obtained with noise that was more complicated than the dichotomous Markovian fluctuations of our system. They also discovered that, for certain types of noise, the flux can actually reverse its direction at some point as noise characteristics are changed. Magnasco focused on time correlations to explain the distinction between equilibrium and non-equilibrium noise [9]. 

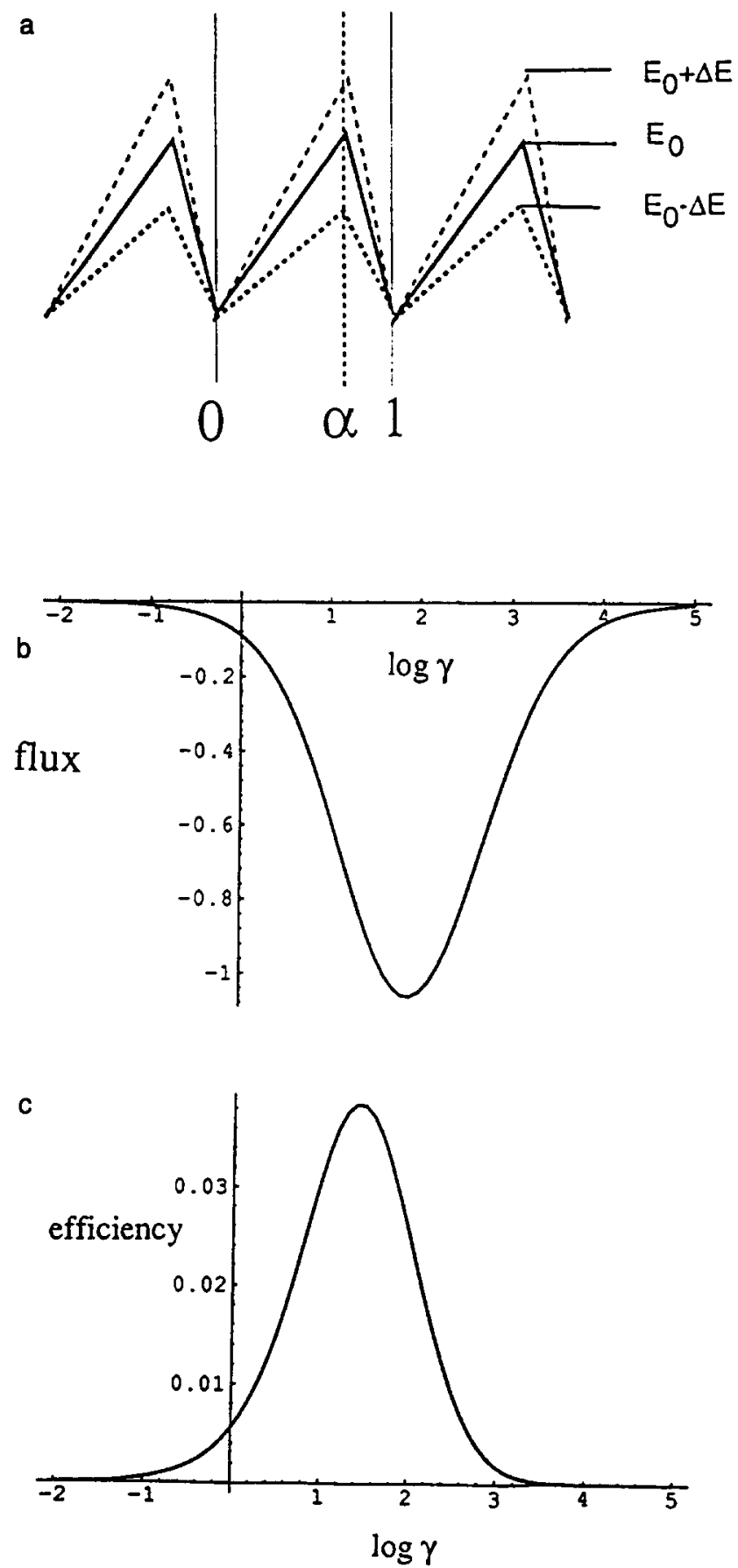

Fig. 3(a) Fokker-Planck equation to describe diffusion on a fluctuating piecewise linear potential can be solved analytically. (b) The flux as a function of the flipping rate $\gamma$. (c) Plot of the efficiency, the calculation of which is explained in the text, as a function of $\gamma$.

An exact solution for Eqs. ( $2 a$ ) and ( $2 b$ ) can be obtained for piecewise linear potentials, as in Fig. 3(a). The potential fluctuates between the $E_{0}+\Delta E$ profile and the $E_{0}-$ $\Delta E$ profile. Fig. 3(b) shows the flux $J$ as a function of the logarithm of the flipping rate $\gamma$. In Ref. [11], we go back to unscaled variables and we take realistic motor protein values: energies of $E_{0} \approx \Delta E \approx 4 k T$; a coefficient of viscous friction of $\beta \approx 6 \times 10^{-6} \mathrm{~s}^{-1}$; and a biopolymer with a period of $80 \AA$ and $\alpha=\frac{7}{8}$. This yields $\gamma \approx 1400$ flips $\mathrm{s}^{-1}$ and a speed of about $100 \mathrm{~nm} \mathrm{~s}^{-1}$. The speed has the correct order of magnitude for motor proteins; flips of the potential profile are supposed to be brought about by the binding of ATP and the release of ADP. A turnover of 700 ATPs per second seems high but small refinements of the model (such as, allowing the rates $\gamma^{+}$and $\gamma^{-}$to be different) can lead to higher speeds at smaller turnovers.

The efficiency of the device can be evaluated and is plotted as a function of $\log \gamma$ in Fig. 3(c). This is the efficiency of the transduction of energy from the non-equilibrium fluctuations to the forward motion of the particle. To bring about the necessary rapid succession of $8 k T$ flips, a chemical conversion with an energy loss much larger than $8 k T$ is required. Therefore, the conversion of chemical energy to forward motion must have an even smaller efficiency than that in Fig. 3(c).

Finally, it is worth noting that, for both $U^{+}$and $U^{-}$, there is no "net tilt" over one period and, in the end, we have flux without a net force. How the fluctuations can end up acting as a macroscopic force $F$ (and $J=F$, because $\beta=1$ ) can be seen in the following way: $J$ is constant along $x$, which implies

$J=\int_{0}^{1} J \mathrm{~d} x$

Substituting $J=J^{+}(x)+J^{-}(x)$ and, subsequently, $J^{ \pm}=$ $\left(F^{ \pm}-\partial_{x}\right) P^{ \pm}$into this integral yields

$$
\langle F\rangle=\langle J\rangle=\int_{0}^{1}\left(F^{+} P^{+}+F^{-} P^{-}\right) \mathrm{d} x
$$

where we have the net force appearing as the average force over the probability distribution. In this picture, flux occurs when, because of the fluctuation, $P^{+}$and $P^{-}$are such that the integral in Eq. (8) gives a non-zero result.

That fluctuations can bring about a macroscopic flux without a net macroscopic force was first recognized about two decades ago, in the general context of stochastic processes, by Tomita and Tomita [11]. They introduced the notions of "irreversible circulation" and "cyclic balance". Only much later was it shown, using chemical kinetics on some simple examples, that the same mechanism could actually drive a reaction against its chemical potential and that oscillations could have the same effect as fluctuations $[12,13]$. It was also pointed out that this could possibly explain how, in living cells, proteins couple reactions, such as how Na, K ATP-ase can use the energy of the ATP turnover to pump sodium and potassium against the chemo-osmotic potential. Replacing an abstract reaction coordinate (which measures the progress of a chemical reaction) by an actual position, taking electrostatic forces instead of thermodynamic forces and with the more fundamental Fokker-Planck formulation instead of chemical kinetics, we arrive at a set-up presented by Ajdari and Prost [14]. They actually built a nanotechnological device that, based on this principle, drove large charged molecules 
[15]. They proposed to develop this further as a method for separation.

\section{A heat engine}

Next, we present an example of a heat engine at a microscopic level. Again with only Fokker-Planck equations as our basis, we show how a temperature gradient can bias Brownian motion. We take the piecewise linear, periodic set-up for the potential as a function of $x$ in Fig. 4 , and derive the flux and the efficiency of the heat engine exactly.

In Fig. 4, region 1 is the interval $\left\langle k, k+\frac{1}{2}\right\rangle$, where $k$ is an integer, and is at temperature $T_{1}$. Region 2 is the interval $\left\langle k+\frac{1}{2}, k+1\right\rangle$ and is at temperature $T_{2}$. If $\left.T_{1}\right\rangle$ $T_{2}$, then it is easier for a particle in the hot region to "jump" over the barrier than it is for a particle in the cold region to do so, and a net flux in the positive direction results. This effect has been studied in great detail by Landauer [16], and his finding that transition rates can be different when a segment along the reaction cordinate is heated up is called the the "blow torch theorem".

In an overdamped medium, and after scaling away the Boltzmann constant $k$ and the coefficient of friction, we have

$$
\begin{array}{ll}
P_{1}^{\prime \prime}(x)+\frac{2 E}{T_{1}} P_{1}^{\prime}(x)=0 & \left(0, \frac{1}{2}\right) \\
P_{2}^{\prime \prime}(x)-\frac{2 E}{T_{2}} P_{2}^{\prime}(x)=0 & \left(\frac{1}{2}, 1\right)
\end{array}
$$

The system is equivalent to a system with a constant temperature $T=1$ and a slope $m_{1}=2 E / T_{1}$ on $\left\langle k, k+\frac{1}{2}\right\rangle$ and slope $m_{2}=-2 E / T_{2}$ on $\left\langle k+\frac{1}{2}, k+1\right\rangle$, leading to a net energy difference of $E\left(T_{1}-T_{2}\right) /\left(T_{1} T_{2}\right)$ over one period. The system is very similar to that studied by Büttiker [17]. However, Büttiker studied a system in which the potential as well as the temperature varied in a sinusoidal manner along the $x$ axis (he actually varied the diffusion coefficient, but this is equivalent to varying the temperature), which leads to more involved algebra than our set-up.

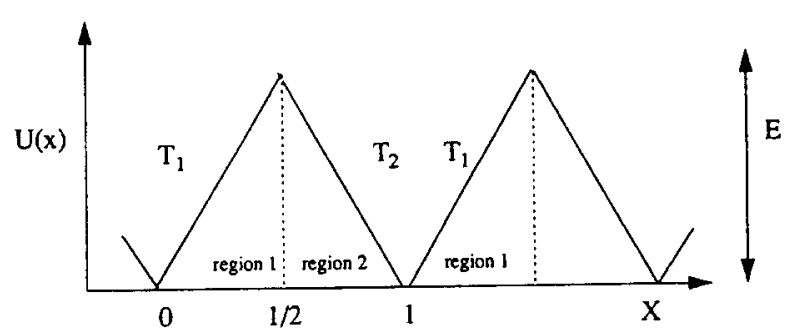

Fig. 4. Piecewise linear potential with the temperature varying along the $x$ axis.
The solution of equations (9a) and (9b) is

$P_{1}(x)=C_{1}+D_{1} \exp \left(-\frac{2 E}{T_{1}} x\right)$

$P_{2}(x)=C_{2}+D_{2} \exp \left[-\frac{2 E}{T_{2}}(1-x)\right]$.

i.e. an exponential "Boltzmann" part plus a constant. The flux is given by

$J_{1}=\left(-\frac{2 E}{T_{1}}-\partial_{x}\right) P_{1}$

$J_{2}=\left(\frac{2 E}{T_{2}}-\partial_{x}\right) P_{2}$.

The exponential "Boltzmann" parts of the probability density distribution in Eq. (10) cancel out when substituted in these expressions. It is the constants that "cause" flux, i.e.

$J_{1}=-\frac{2 E C_{1}}{T_{1}}$

$J_{2}=\frac{2 E C_{2}}{T_{2}}$.

Demanding that the probability density and the flux are continuous at the corner points between regions 1 and 2 (i.e. $P_{1}(0)=P_{2}(1), P_{1}(1 / 2)=P_{2}(1 / 2)$ and $\left.J_{1}=J_{2}\right)$ and normalizing the total probability over one period to unity (i.e. $\int_{0}^{1} P(x) \mathrm{d} x=1$ ) leads to equations from which we can solve for $C_{1}, D_{1}, C_{2}$ and $D_{2}$. With

$$
\begin{aligned}
R= & E\left[\exp \left(E / T_{1}\right)-\exp \left(E / T_{2}\right)\right]\left(T_{1}-T_{2}\right) \\
& +\left[\exp \left(E / T_{1}\right)-1\right]\left[\exp \left(E / T_{2}\right)-1\right]\left(T_{1}+T_{2}\right)^{2}
\end{aligned}
$$

we have

$$
\begin{aligned}
& C_{1}=\frac{2\left[\exp \left(E / T_{1}\right)-\exp \left(E / T_{2}\right)\right] E T_{1}}{R} \\
& D_{1}=\frac{2 \exp \left(E / T_{1}\right) E\left[\exp \left(E / T_{2}\right)-1\right]\left(T_{1}+T_{2}\right)}{R} \\
& C_{2}=\frac{-2\left[\exp \left(E / T_{1}\right)-\exp \left(E / T_{2}\right)\right] E T_{2}}{R} \\
& D_{2}=\frac{2 \exp \left(E / T_{2}\right) E\left[\exp \left(E / T_{1}\right)-1\right]\left(T_{1}+T_{2}\right)}{R}
\end{aligned}
$$

For $T_{1}=1.05$ and $T_{2}=0.95$, Fig. 5(a) shows the flux as a function of the barrier height $E$. Symmetry does not allow for any flux at $E=0$. For $E \rightarrow \infty$, no particle will ever mount the barrier at a finite temperature. In between, for a finite value of $E$, the flux has a maximum. A similar graph would be seen if, instead of the barrier height, the temperature of both regions were changed by the same multiplicative factor; maxima that have been found with 
variation of the temperature (or, equivalently, input noise density) have been called "stochastic resonance" [18].

An important issue is also the efficiency of this engine.
The upper limit for the efficiency, of course, is the Carnot efficiency $\left(T_{1}-T_{2}\right) / T_{1}$. Next, we will calculate the actual efficiency of this engine.

a

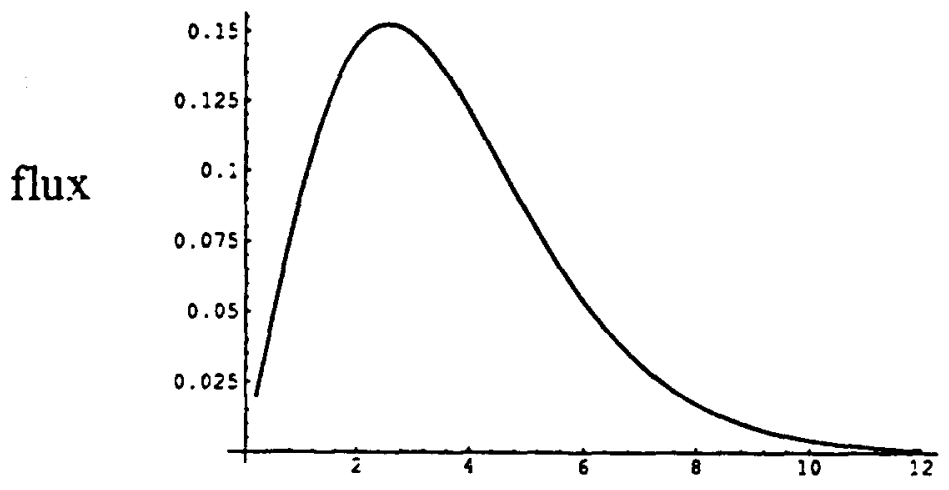

E

b

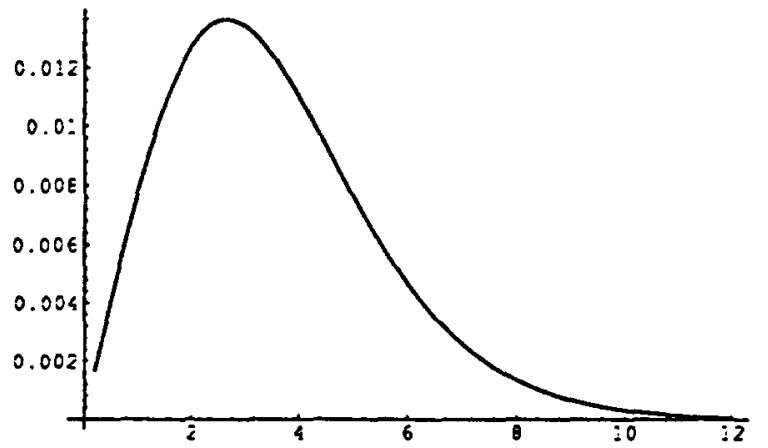

$\mathrm{E}$

C

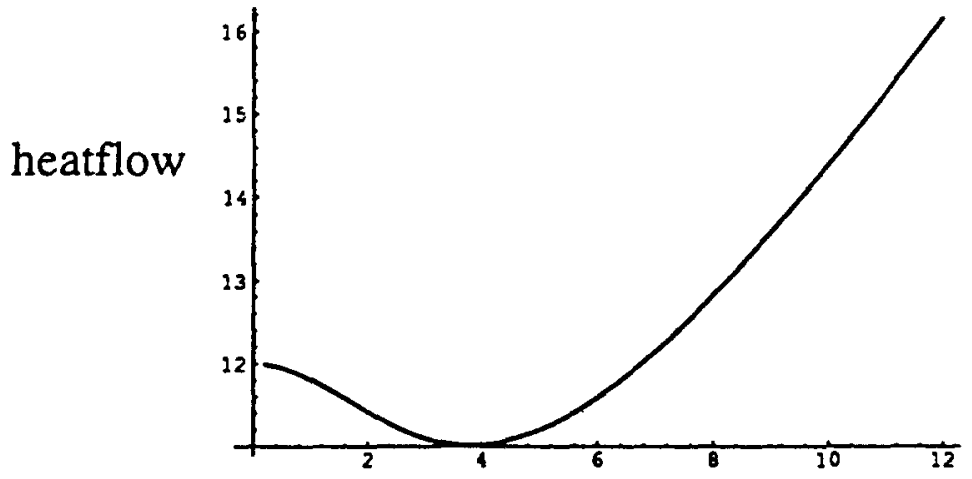

$\mathbf{E}$

Fig. 5(a) The flux and (b) the efficiency as functions of the barrier height $E$ for the analytically solvable, piecewise linear heat engine that is explained in the text. The flux and the efficiency are not simple multiples of one another; their ratio is the heat flow into the system that is necessary to keep it at stationarity, as depicted in $2(c)$. 
Whenever particles enter region 1 , they have to be heated to maintain stationarity. However, the net flux is not only the inflow into region 1; it can actually be calculated as the difference between the forward and backward flows at both the corner points and, as such, it is only a fraction of the total inflow into region 1 . This fraction is the efficiency of the engine.

For a particle at position $x$ in an interval with two absorbing barriers, it is possible to calculate the probabilities for the particle eventually to exit at the right-hand vs. at the left-hand barrier (see Ref. [3], p. 142). Applying this to our system, we find that a particle at $x$ in $\left\langle 0, \frac{1}{2}\right\rangle$ has a probability of exiting over the maximum at $x=\frac{1}{2}$ of

$\pi_{1}^{(1 / 2)}(x)=\frac{\exp \left(2 E x / T_{1}\right)-1}{\exp \left(E / T_{1}\right)-1}$

For the probability that a particle at $x$ in $\left\langle\frac{1}{2}, 1\right\rangle$ exits over the maximum at $x=\frac{1}{2}$, we have

$\pi_{2}^{(1 / 2)}(x)=\frac{\exp \left[2 E(1-x) / T_{2}\right]-1}{\exp \left(E / T_{2}\right)-1}$

Of course, we have for the exiting probabilities at the bottom that

$\pi_{1}^{(0)}(x)=1-\pi_{1}^{(1 / 2)}(x)$

$\pi_{2}^{(1)}(x)=1-\pi_{2}^{(1 / 2)}(x)$

The probability for a particle to be in $\left(0, \frac{1}{2}\right)$, i.e. region 1 , where the temperature is $T_{1}$, is

$p_{1}=\int_{0}^{1 / 2} P_{1} \mathrm{~d} x=\frac{1}{2}\left\{C_{1}+\frac{D_{1} T_{1}}{E}\left[1-\exp \left(-\frac{E}{T_{1}}\right)\right]\right\}$

and, likewise, we have $p_{2}=1-p_{1}$. Next, we define

$\tilde{P}_{1}(x)=\frac{1}{p_{1}}\left[C_{1}+D_{1} \exp \left(-\frac{2 E x}{T_{1}}\right)\right]$

which is the probability density of a particle to be at $x$, given that that particle is in region 1. Similarly, we have

$\tilde{P}_{2}(x)=\frac{1}{p_{2}}\left\{C_{2}+D_{2} \exp \left[-\frac{2 E(1-x)}{T_{2}}\right]\right\}$

The probability that an average particle in region 1 exits at $x=\frac{1}{2}$ is given by

$p_{12}^{(1 / 2)}=\int_{0}^{1 / 2} \pi_{1}^{(1 / 2)} \tilde{P}_{1} \mathrm{~d} x$

and, for an average particle in region 2 , the probability for escape at $x=\frac{1}{2}$ is

$p_{21}^{(1 / 2)}=\int_{1 / 2}^{1} \pi_{2}^{(1 / 2)} \tilde{P}_{2} \mathrm{~d} x$

These quantities allow us then to evaluate the efficiency.

The probability density is stationary; thus, for every $N$ particles that leave region 1 for region 2, there are also $N$ particles that flow from region 2 to region 1 . Of the $N$ particles that flow from region 1 to region 2, an amount $N p_{12}{ }^{(1 / 2)}$ moves over the maximum at $x=\frac{1}{2}$. However, of the particles that flow from region 2 to region 1 , an amount $N p_{21}{ }^{(1 / 2)}$ moves over the $x=\frac{1}{2}$ maximum. This implies a net transfer of $N\left(p_{12}{ }^{(1 / 2)}-p_{21}{ }^{(1 / 2)}\right)$ by one period in the positive direction. With Eqs. (15)-(19) it is possible to express the efficiency in terms of $C_{1}, D_{1}, C_{2}$, $D_{2}, E, T_{1}$ and $T_{2}$. Using Eq. (14), it is even possible to go back to just $E, T_{1}$ and $T_{2}$. The formulae in that case become very long and cumbersome, but are easily handled with any computer algebra system.

Fig. 5(b) shows the efficiency as a function of $E$ at $T_{1}=0.95$ and $T_{2}=1.05$. It appears that the efficiency never reaches more than about $15 \%$ of the Carnot efficiency.

Although a brief glance at Figs. 5(a) and 5(b) suggests that the flux and the efficiency are simple multiples of one another, upon quantitative evaluation, it appears that the flux/efficiency ratio is not constant. This ratio actually gives the amount of heat that has to be pumped into the system per unit of time to keep it stationary, and is depicted in Fig. 5(c). In the $E \rightarrow \infty$ limit, the probability density distributions on regions 1 and 2 become delta functions in the same point and an infinite amount of heat exchange results.

Feynman's ratchet-and-pawl device [5] has two separate reservoirs that are at different temperature and are each at equilibrium. One reservoir contains the end with the vanes, while the other reservoir contains the ratchet and pawl. The only way in which the reservoirs exchange energy is through the action of the device. Therefore, it is no surprise that the maximum theoretical Carnot efficiency is attained. However, Feynman's device is a very artificial one and mixes macroscopic (isolated reservoirs and a solid device with rotating parts, etc.) and microscopic (Brownian) physics - it is a "thought experiment" more than anything else. Our more realistic "Fokker-Planck" heat engine is also more inefficient: heat is flowing, even at $E=0$ or $E \rightarrow \infty$ when there is no flow and no net work is being done.

\section{Discussion}

We have shown above that it is possible to bias Brownian motion with the input of other forms of energy. A feature of both the engines that we presented is the low efficiency. For the chemical engine, it is important to realize that, in an overdamped medium, the power that is dissipated by a particle moving with velocity $v$ is $\beta v^{2}$. This means that the most efficient way to cover a certain distance $L$ in a time $T$ is to move with a constant speed $L / T$. Any variation in speed implies that more energy is needed to cover the distance $L$. We calculated $P_{\text {out }}$ as the power that would be necessary to make the motor move at a constant speed. 
Going back to a Langevin picture, we can understand how, in the model of Fig. 3, the fluctuations cause a lot of motion within a well before a "jump" is made. This stepwise motion is also what is experimentally observed on actual motor proteins [19]. Because of its stepwise nature, any fluctuation-driven motion in an overdamped medium is bound to be very inefficient. Using chemical kinetics, efficiencies have been calculated for the fluctuation-mediated transduction of energy from the breakdown of one chemical (S) to the synthesis of another chemical (P) [20]. Larger efficiencies can then be achieved, because the process has an entirely different character. Power is not dissipated as $\beta v^{2}$ but, instead, is stored at a rate of $P_{\text {out }}=\beta^{\prime} v$, where $v$ is now the rate of production of chemical P. Because of the linearity in $v$, the character of the process, i.e. smooth vs. stepwise, does not affect the amount of energy needed to synthesize a certain amount of chemical $\mathbf{P}$.

For motor proteins, it has been experimentally shown that the coefficient of friction does not arise from the viscosity of the solution but from the "friction" on the connection between the motor protein and the biopolymer. It is unlikely that this friction can be thought of as constant over the whole period along the biopolymer. For non-constant $\beta(x)$, a constant velocity is no longer the most efficient way of moving and the $v(x)$ that gives maximal efficiency can be easily calculated. The observed stepwise motion is efficient when the coefficient of friction is high in the region where the protein remains "stuck" for a while and low in the region that is "stepped over."

For a single kinesin molecule providing internal transport in a cell, the efficiency is not an issue, because the power involved is negligible compared with that transduced in other processes in the cell. In muscle, however, the action of motor proteins involves a much greater part of the energy budget and efficiency does become important. The efficiency can then be increased by adding small features to make it look increasingly more like a RubeGoldberg device. Actual motor proteins are observed to be connected to the bioploymer with two "legs." Peskin et al. showed how two coupled Brownian particles that are diffusing on fluctuating potentials can "drag" one another along with an elastic chain, so making the motion smoother and, thus, possibly achieving greater efficiency [21]. However, these designs can only be analyzed through numerical simulation, and the chemical engine described above still holds the underlying principle. To our knowledge, the piecewise linear potential that we discussed is the only case that can easily be evaluated analytically.

The heat engine is of lesser biological significance. It is generally believed that organisms function isothermally. However, it has been conjectured that, in environments such as convection currents in natural waters, bacteria can evolve that employ mechanisms to build up an ATP-ADP gradient, deriving energy from thermal cycling [22].

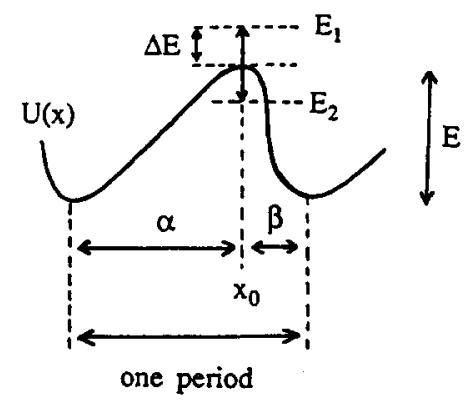

Fig. 6. A potential with a maximum that fluctuates between $E_{1}$ and $E_{2}$.

In both systems that we have studied, the noise (non-zero temperature) is essential to achieve the flux. At zero temperature, both systems come to a standstill and, if the temperature becomes too high, the energy profile $U(x)$ becomes relatively insignificant and the flux again vanishes. These characteristics apply to our system as well as to the double well in Ref. [18], by means of which McNamara and Wiesenfeld explained the "stochastic resonance" phenomenon. It was found that, when the energy difference between the wells is oscillating, the flux between the wells peaks for some finite value of the temperature. With some brief heuristic arguments, we will show that, in our system as well as in the double well of Ref. [18], the flux as a function of the temperature has a shape given by

$J \approx \frac{1}{T^{a}} \exp \left(-\frac{A}{T}\right)$

where $A>0$ and $a>0$. This is also the form of the formula derived in Ref. [18] for the signal-to-noise ratio in the oscillating double well.

Let us consider the fluctuating periodic potential in Fig. 6 . The characteristic times for relaxation to adiabaticity on the long and short slopes have a ratio $\tau_{\alpha} / \tau_{\beta}=(\alpha / \beta)^{2}$. We take the flipping time to be the geometric average of $t_{\alpha}$ and $t_{\beta}$, i.e. $t_{\text {flip }}=\left(t_{\alpha} t_{\beta}\right)^{1 / 2}$, and then assume that we have instantaneous adiabatic adjustment on the short slope and a Boltzmann distribution on the average potential on the long slope. This actually brings about discontinuities at the borders between the regions, which would not occur in a rigorous treatment.

When we next assume that, for the flow over the top, we have

$$
\begin{aligned}
J_{\alpha \rightarrow \beta} & =C^{\prime} \lim _{x \uparrow x_{0}} P_{\alpha}(x)=C \exp \left[-\frac{\langle U(x)\rangle}{k T}\right] \\
& =C \exp \left(-\frac{E_{1}+E_{2}}{2 k T}\right) \\
J_{\beta \rightarrow \alpha} & =C^{\prime} \lim _{x \downarrow x_{0}} P_{\beta}(x)=C\left\langle\exp \left[-\frac{U(x, t)}{k T}\right]\right\rangle \\
& =C \frac{1}{2}\left[\exp \left(-\frac{E_{1}}{k T}\right)+\exp \left(-\frac{E_{2}}{k T}\right)\right]
\end{aligned}
$$


where $C$ and $C^{\prime}$ are proportionality constants, and the energy is no longer in units of $k T$, we obtain for the net flow that

$$
\begin{aligned}
J_{\text {net }} & =J_{\alpha \rightarrow \beta}-J_{\beta \rightarrow \alpha} \\
& \approx-\left[\exp \left(\frac{E_{1}}{k T}\right)+\exp \left(\frac{E_{2}}{k T}\right)-2 \exp \left(\frac{E_{1}+E_{2}}{2 k T}\right)\right]
\end{aligned}
$$

which is always negative. For small $\Delta E$, this can be approximated by

$J_{\text {net }} \approx\left(\frac{\Delta E}{k T}\right)^{2} \exp \left(-\frac{E}{k T}\right)$

For the heat engine, we take $T_{1}=T(1+\varepsilon)$ and $T_{2}=$ $\mathrm{T}(1-\varepsilon)$, assume a Boltzmann distribution on each leg, i.e.

$P_{1}(x) \approx\left(\frac{E}{T}\right) \exp \left(\frac{-E}{T_{1}}\right)$

$P_{2}(x) \approx\left(\frac{E}{T}\right) \exp \left(\frac{-E}{T_{2}}\right)$

for small $\varepsilon$, and again take for the flow over the top that

$$
\begin{aligned}
J_{\text {net }} & =J_{1 \rightarrow 2}-J_{2 \rightarrow 1} \approx \lim _{x \uparrow 1 / 2} P_{1}(x)-\lim _{x \downarrow 1 / 2} P_{2}(x) \\
& =\frac{E}{T} \exp \left(-\frac{E}{T}\right)\left[\exp \left(\frac{E \varepsilon}{T}\right)-\exp \left(-\frac{E \varepsilon}{T}\right)\right]
\end{aligned}
$$

At the first order in $\varepsilon$, this reduces to

$$
J_{\mathrm{net}} \approx \varepsilon\left(\frac{1}{T}\right)^{2} \exp \left(\frac{-E}{T}\right),
$$

The $\exp (-A / T)$ terms in these formulae give the rate at which a large central barrier is crossed - a barrier that could never be crossed in the absence of noise. The $\varepsilon / T^{a}$ term is a consequence of the fact that the system is asymmetric, in the sense that a left-to-right look at the profile differs from a right-to-left look. The oscillating double well of Ref. [18] is symmetric around the central barrier, in the sense that, during one period of the oscillation, there is as much flow from left to right as there is from right to left. However, the same ideas apply to the amplitude of the oscillating flux $J$ over the central barrier, and a formula of a form similar to Eq. (20) can be derived for this amplitude. With the development of nanotechnology and the theoretical and experimental studies of biological systems on a molecular level, the future might hold an abundance of variations on this theme in store.

\section{Acknowledgments}

This work was supported in part by NIH Grant R010ES06010. We acknowledge Charles Doering, Armand Ajdari, Thomas Witten and Carey Bagdassarian for useful discussions.

\section{References}

[1] Webster's Collegiate Dictionary, Merriam-Webster Inc., Springfield MA, 10th edn., 1993.

[2] J. Darnell, H. Lodish and D. Baltimore, Molecular Cell Biology, Scientific American Books, New York, 1990, Ch. 17-19.

[3] C.W. Gardiner, Handbook of Stochastic Methods, Springer, Berlin, 2nd edn., 1985

[4] E.W. Taylor, Nature, 361 (1993) 115-116.

[5] R.P. Feynman, R.B. Leighton and M. Sands, The Feynman Lectures on Physics, Vol. 1, Addison Wesley, Reading, MA, 1966, Ch. 46.

[6] L. Brillioun, Phys. Rev. 78 (1950) 627.

[7] C.R. Doering, W. Horsthemke and J. Riordan, Phys. Rev. Lett., 72 (1994) 2984-2987.

[8] M.M. Millonas and M.I. Dykman, Phys. Lett. A, 185 (1994) 65-69.

[9] M. Magnasco, Phys. Rev. Lett., 71 (1993) 1477-1481.

[10] R.D. Astumian and M. Bier, Phys. Rev. Lett., 72 (1994) 1766-1769.

[11] K. Tomita and H. Tomita, Prog. Theor. Phys., 51 (1974) 1731

[12] T.Y. Tsong and R.D. Astumian, Bioelectrochem. Bioenerg., 15 (1986) 457-476.

[13] R.D. Astumian, P.B. Chock, T.Y. Tsong, Y.-D. Chen and H.V. Westerhoff, Proc. Natl. Acad. Sci. U.S.A., 84 (1987) 434-438.

[14] A. Ajdari and J. Prost, C.R. Acad. Sci. Paris, 315 (1992) 1635-1639.

[15] J. Rousselet, L. Salome, A. Ajdari and J. Prost, in Nature submitted for publication.

[16] R. Landauer, J. Stat. Phys., 53 (1988) 233-248.

[17] M. Büttiker, Z. Phys. B, Cond. Matter, 68 (1987) 161-167.

[18] B. McNamara and K. Wiesenfeld, Phys. Rev. A, 39 (1994) 48544869.

[19] K. Svoboda, C.F. Schmidt, B.J. Schnapp and S.M. Block, Nature, 365 (1993) 721-727.

[20] R.D. Astumian, P.B. Chock, T.Y. Tsong and H.V. Westerhoff, Phys, Rev. A, 39 (1989) 6416-6435.

[21] C.S. Peskin, G. Bard Ermentrout and G. Oster, in V. Mow et al. (eds.), Cell Mechanics and Cellular Engineering, Springer, Berlin, 1994.

[22] A.W.J. Muller, Chem. Physiol. Med. NMR, 25 (1993) 95-111. 